\title{
Softening, localization and stabilization: capture of discontinuous solutions in J2 plasticity.
}

\author{
M. Cervera, M. Chiumenti and C. Agelet de Saracibar \\ International Center for Numerical Methods in Engineering (CIMNE) \\ Technical University of Catalonia (UPC)
}

Edificio C1, Campus Norte, Jordi Girona 1-3, 08034 Barcelona, Spain.

KEYWORDS: incompressibility, plasticity, softening, localization and stabilization.

\begin{abstract}
This paper exploits the concept of stabilization techniques to improve the behaviour of mixed linear/linear simplicial elements (triangles and tetrahedra) in incompressible or nearly incompressible situations. Elasto-J2-plastic constitutive behaviour has been considered with linear and exponential softening. Two different stabilization methods are used to attain global stability of the corresponding discrete finite element formulation. Implementation and computational aspects are also discussed, showing that a robust application of the proposed formulation is feasible. Numerical examples show that the formulation derived is free of volumetric locking and spurious oscillations of the pressure, and also that the results obtained are practically mesh independent, comparing very favourably with those obtained with the standard, non-stabilized, approaches.
\end{abstract}




\section{Introduction}

Softening materials subjected to monotonic straining exhibit strain localization. The nature of this localization depends on the particular behaviour of the material. In the so-called J2 materials, shear (or slip) strains concentrate; in Rankine-type materials, only normal elongations localize. This phenomenon leads to the formation of localization bands inside the solid where, once the peak stress is reached, the deformation concentrates while the material outside the band unloads elastically. This induces the formation of weak discontinuities, with continuous fields of displacements but discontinuous fields of strain inside and outside the bands (see Figure 1a).

Upon continuing straining, the width of the localization band diminishes and, unless there is a physical limitation, it tends to zero. Ultimately, this process may lead to strong discontinuities, with discontinuous fields of displacements across the discontinuity line, and unbounded strains (see Figure 1b). In J2 materials, these are called slip lines. It is generally accepted that the amount of energy released during the formation of a unit length of
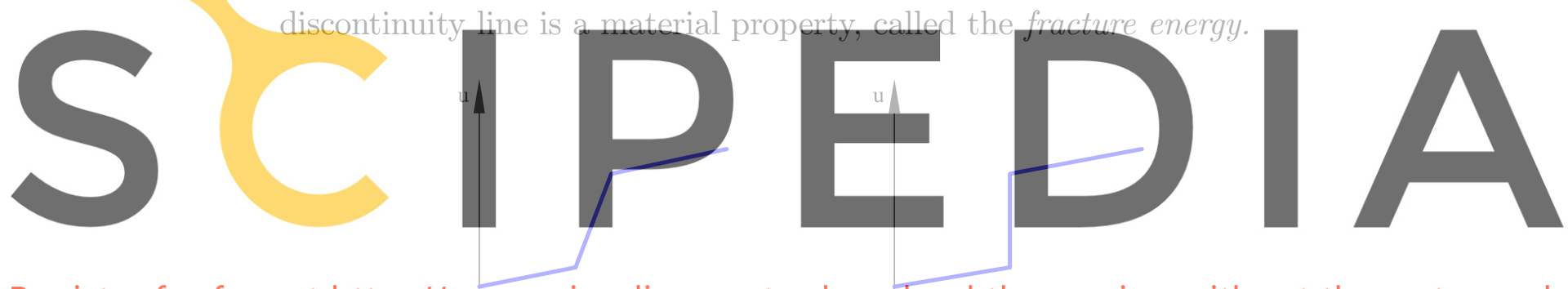

Register for free at https//wWw.scipedia.comto download the version without the watermark

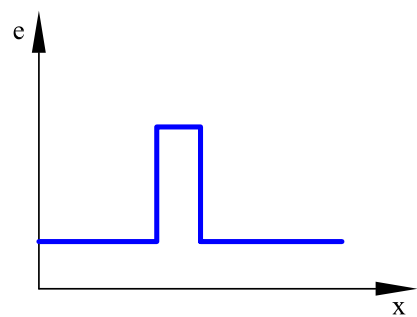

a)

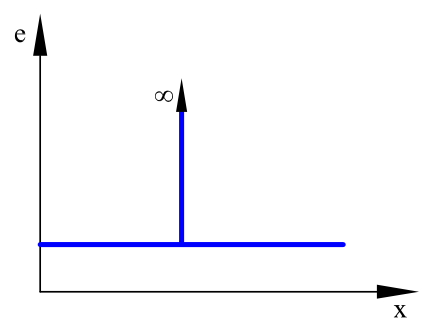

b)

Figure 1: Strain localization: (a) weak discontinuity; (b) strong discontinuity 
In the last two decades, many different finite element strategies have been devised to model discontinuities and the references in the bibliography are innumerable. Two comprehensive and up-to-date overviews are given in references [1] and [2]. The possibilities are several, and both the weak and the strong discontinuity approaches have been followed. In the first, the objective is to capture the localization band as precisely as possible, with standard continuous elements (see, for instance, reference [3]). In the second, the displacement field is enhanced with discontinuous functions so that the true discontinuity line can be captured (see, for instance, reference [4]). It is always possible to interpret a weak discontinuity as a regularization of a strong one, with the discontinuity "smeared" across the maximum possible resolution of the mesh, that is, one element.

Remarkably, most of the studies about localization with J2 plasticity have been carried out using the irreducible formulation, with the displacement field as only unknown variable. Unfortunately, J2 plastic flow is isochoric, and the irreducible formulation is not well suited to cope with the corresponding incompressibility constraint. Even for compressible elastic materials, for
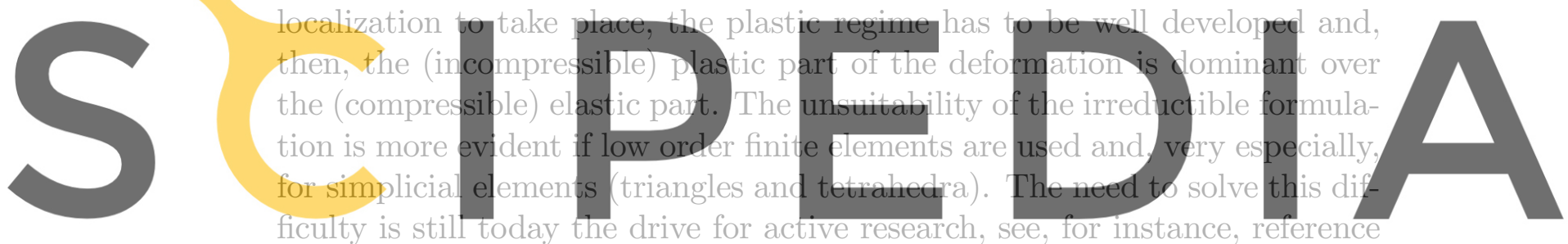
Register for free at

the appropriate framework to tackle (quasi)-incompressible problems [6]. In fact, very promising results have been obtained in localization problems with J2 plasticity using this formulation together with remeshing techniques in [7] and [8] and in coupled dynamic problems in [9] and [10]. Nevertheless, it is very difficult to construct stable low order elements and, again, particularly, low order simplicial elements. This is another very attractive area of ongoing research, see references [11], [12], [13], [14], [15], [16], [17], [18], [19], [20], among others.

Recently, the authors have applied stabilization methods, originated in the fluid dynamics community ([21], [22], [23] and [24]), to the solution of incompressible elasto-plastic problems with mixed linear/linear simplicial elements, see [25], [26] and [27]. In this paper, we extent the applicability of these stabilization procedures to the softening plastic regime, to show that 
results can be obtained which are free of pressure oscillations and volumetric locking and, also, which are practically mesh independent. This translates in the achievement of two important goals: (a) the position and orientation of the localization band is independent of the directional bias of the finite element mesh and (b) the global post-peak load-deflection curves are independent of the size of the elements in the localization band.

The accomplishment of these objectives is attained by ensuring both global and local stability of the problem. Global stability is necessary to preclude global wild oscillations of the pressure induced by the incompressible behaviour inside the localization band. In this work, this is secured by the modification of the standard Galerkin variational formulation, making use of the concept of sub-grid approximation. On the other hand, local stability is necessary to eliminate local oscillations in the vicinity of the band. Here, this is fulfilled by a slight modification of the global stabilization procedure, namely, making the stabilization parameter depending on the amount of plastic deformation accumulated inside the localization band. This results in a suitable stabilization technique, virtually free of spurious pressure
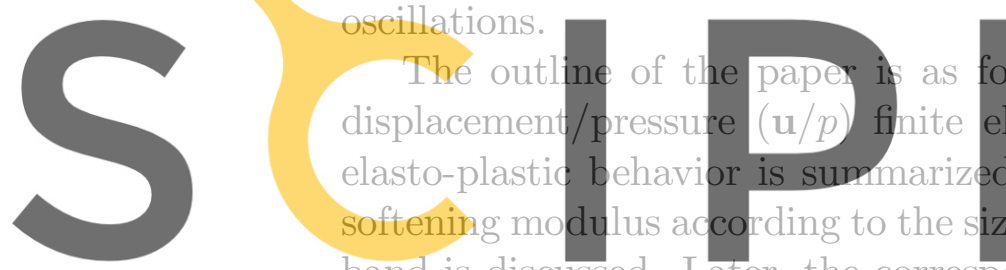

band is discussed. Later, the corres
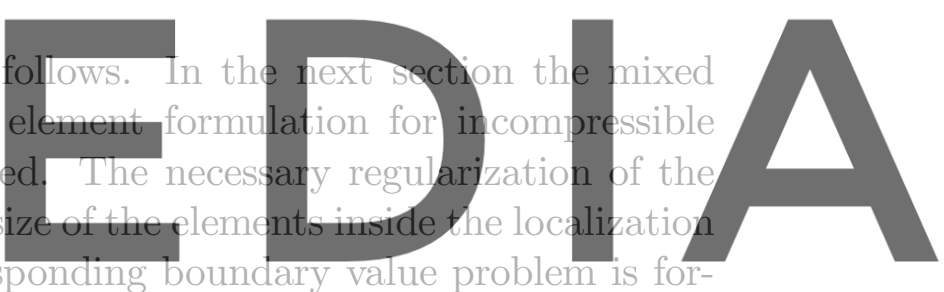

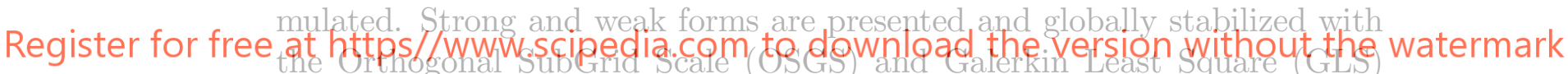

methods. Also, a nonlinear modification of the stabilization parameter is in-

troduced to get rid of the local oscillations in the neighbourhood of the localization band. The possibility of enhancing the convergence of the numerical procedure by the inclusion of artificial viscosity, in a consistent fashion, is also considered. Implementation and computational aspects are discussed next. Finally, some numerical benchmarks and examples are presented to assess the present formulation and to compare its performance with the standard irreductible and mixed Galerkin triangular and tetrahedral elements. 


\section{Mixed formulation for J2 plasticity and vis- coplasticity}

\section{$2.1 \quad J 2$ plasticity and viscoplasticity}

The formulation of the incompressible elasto-plastic mechanical problem can be written considering the hydrostatic pressure $p$ as an independent unknown, additional to the primary displacement field. The stress tensor $\boldsymbol{\sigma}$ is then expressed as:

$$
\boldsymbol{\sigma}=p \mathbf{1}+\mathbf{s}
$$

where $p=\frac{1}{3} \operatorname{tr}(\sigma)$ and $\mathbf{s}=\operatorname{dev}(\sigma)$ are the volumetric and the deviatoric parts of the stress tensor, respectively. Correspondingly, the strain tensor $\varepsilon=\nabla^{s} \mathbf{u}$, where $\mathbf{u}$ are the displacements, is expressed as:

$$
\varepsilon(\mathbf{u})=\frac{1}{3} \varepsilon_{v} \mathbf{1}+\mathbf{e}
$$
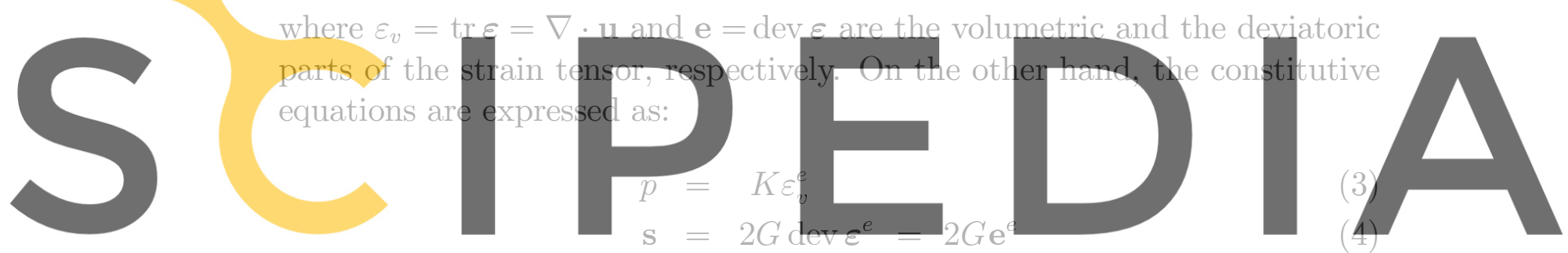

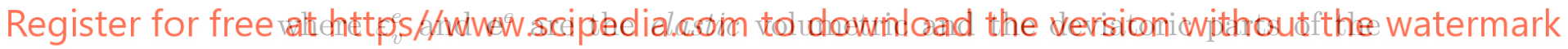

strain tensor, respectively; $K$ is the bulk modulus, also referred to as modulus

of volumetric compressibility, and $G$ is the shear modulus. In incompressible elasticity, $K$ tends to infinity and, thus, $\varepsilon_{v}^{e}$ vanishes. Additionally, in incompressible (J2) plasticity, the volumetric part of plastic deformation is zero, so that $\varepsilon_{v}=\varepsilon_{v}^{e}=\nabla \cdot \mathbf{u}=0$. On the other hand, the elastic deviatoric strain tensor $\mathbf{e}^{e}$ is defined as:

$$
\mathbf{e}^{e}=\mathbf{e}-\mathbf{e}^{p}
$$

where $\mathbf{e}^{p}$ is the plastic strain tensor, which in $\mathrm{J} 2$ plasticity is purely deviatoric.

Box 1 summarizes the elasto-plastic model used in this work, accounting for isotropic softening. As usual, the equivalent plastic strain is defined as $\xi=$ $(\sqrt{2 / 3}) \int_{o}^{t}\left\|\dot{\mathbf{e}}^{p}\right\| d t$, and the equivalent von Mises stress is $\bar{s}=(\sqrt{3 / 2})\|\mathbf{s}\|$. With these definitions, the rate of plastic work is $\dot{\mathcal{W}}^{p}=\mathbf{s}: \dot{\mathrm{e}}^{p}=\bar{s} \dot{\xi}$. 
Notice in Box 1 that the isotropic softening variable $r=r(\xi)$ defines the current size of the yield surface, as it controls the value of the radius of the von Mises cylinder. Initially, when the equivalent plastic strain $\xi=0, r$ is equal to the initial flow stress $\sigma_{o}$. Along the softening regime, $r$ diminishes and, for large value of the equivalent plastic strain, it vanishes.

The plastic multiplier $\dot{\gamma}$ is determined from the Kuhn-Tucker and consistency conditions:

$$
\begin{aligned}
& \dot{\gamma} \geq 0 \quad \Phi(\mathbf{s}, r) \leq 0 \quad \dot{\gamma} \Phi(\mathbf{s}, r)=0 \\
& \text { if } \Phi(\mathbf{s}, r)=0 \text { then } \dot{\gamma} \dot{\Phi}(\mathbf{s}, r)=0
\end{aligned}
$$

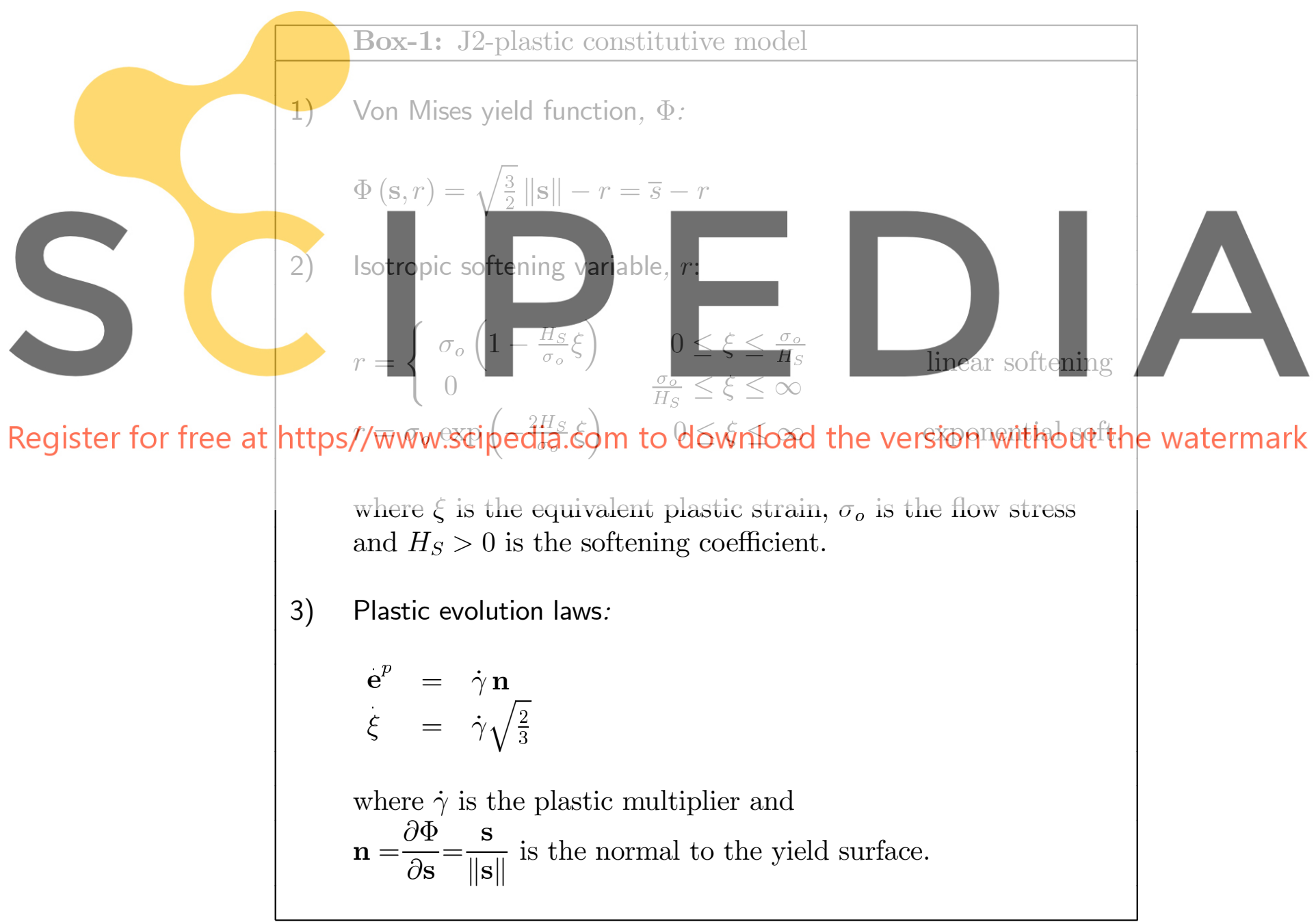


The elasto-plastic model described so far can be considered as the limiting inviscid case of a rate-dependent J2 elasto-viscoplastic model. The only formal difference between viscoplasticity and inviscid plasticity is that in the viscous case the Kuhn-Tucker and consistency conditions are replaced by an explicit evolution law for the plastic multiplier $\dot{\gamma}$, for instance in the form:

$$
\dot{\gamma}=\left\langle\frac{\Phi}{\eta}\right\rangle^{m}
$$

where $\eta$ is the viscosity and $m$ is a viscosity exponent. Notice that for very low values of the viscosity, $\eta \rightarrow 0$, or very small relaxation times, $\bar{\tau}:=\eta / G$, the inviscid plastic model is recovered.

Details on how to integrate along time both the elasto-plastic and the elasto-viscoplastic constitutive models can be found in [28]. As it will be explained later, it is often advantageous to use a consistent viscous regularization of the plastic case to be able to obtain solutions for the inviscid case in a robust and efficient way.
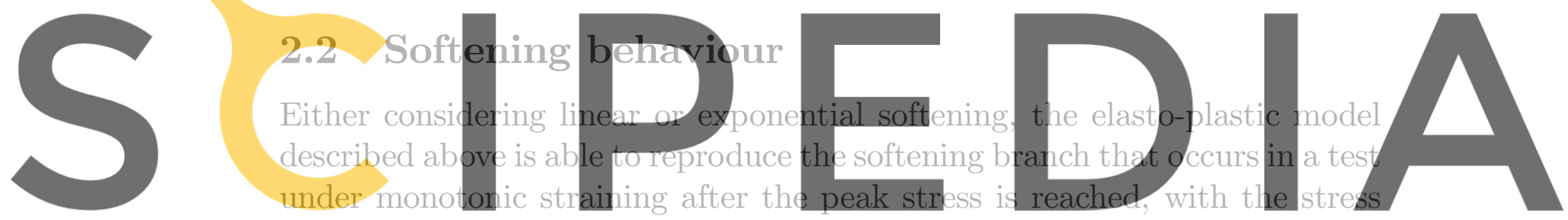

decreasing asymptotically to the strain axis. With such behaviour, a finite

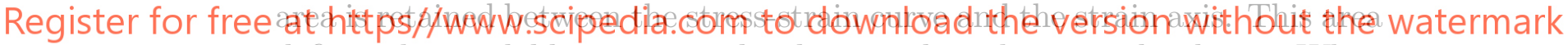
defines the available energy to be dissipated in the control volume. When modeling weak discontinuities (or regularized strong discontinuities) with finite elements, this energy has to be appropriately related to the fracture energy of the material, $G_{f}$, to satisfy the requisites of mesh-size objectivity. This is accomplished by the introduction of a geometrical factor, $l_{\mathrm{ch}}$, called characteristic length, which depends on the spatial discretization and ensures conservation of the energy dissipated by the material upon mesh-size refinements [3]. Accordingly, the determination of the softening parameter $H_{S}$ is made by equating the material fracture energy per unit of characteristic length to the total dissipation along the deformation process.

For the elasto-plastic model, the rate of plastic work can be computed as:

$$
\dot{\mathcal{W}}^{p}=\mathbf{s}: \dot{\mathbf{e}}^{p}=\bar{s} \dot{\xi}=r(\xi) \dot{\xi}
$$


Thus, the total plastic work along a process with softening is

$$
\mathcal{W}^{p}=\int_{t=0}^{t=\infty} \dot{\mathcal{W}}^{p} d t=\int_{\xi=0}^{\xi=\infty} r(\xi) \dot{\xi}=\frac{\sigma_{o}^{2}}{2 H_{S}}
$$

It has to be remarked that the total plastic work is equal to the "area" below the $r-\xi$ curve, that defines the softening response. Note also that the result in Eq. (9) holds both for linear and exponential softening, due to the appropriate definition of these in Box 1.

Equating the total plastic work to the energy (per unit volume) to be dissipated in the localization band, $\mathcal{W}^{p}=G_{f} / l_{\mathrm{ch}}$, it yields:

$$
H_{S}=\frac{\sigma_{o}^{2}}{2 G_{f}} l_{\mathrm{ch}}=\bar{H}_{S} l_{\mathrm{ch}}
$$

where $\bar{H}_{S}$ only depends on the material properties. This is consistent with the results obtained in the strong discontinuity approach [4]. For linear elements, the characteristic length can be taken as the representative size of
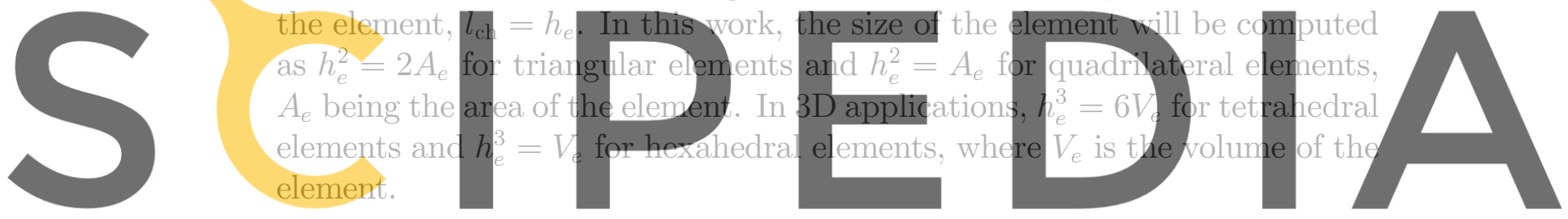

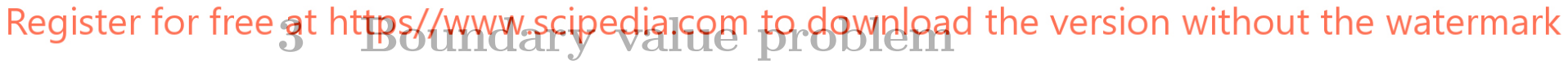

\subsection{Strong and weak forms}

The strong form of the continuum mechanical problem can be stated as: find the displacement field $\mathbf{u}$ and the pressure field $p$, for given prescribed body forces $\mathbf{f}$, such that:

$$
\begin{aligned}
\nabla \cdot \mathbf{s}+\nabla p+\mathbf{f} & =\mathbf{0} & & \text { in } \Omega \\
\nabla \cdot \mathbf{u}-\frac{1}{K} p & =0 & & \text { in } \Omega
\end{aligned}
$$

where $\Omega$ is the open and bounded domain of $\mathbb{R}^{n_{\operatorname{dim}}}$ occupied by the elastoplastic solid in a space of $n_{\text {dim }}$ dimensions. Eqs. (11)-(12) are subjected to appropriate Diritchlet and Neumann boundary conditions. In the following, 
we will assume these in the form of prescribed displacements $\mathbf{u}=\overline{\mathbf{u}}$ on $\partial \Omega_{u}$, and prescribed tractions $\overline{\mathbf{t}}$ on $\partial \Omega_{t}$, respectively. In the mixed formulation the value of the pressure is defined by the Neumann conditions or, alternatively, by prescribing its value at some point.

The associated weak form of the problem $(11)+(12)$ can be stated as:

$$
\begin{aligned}
(\mathbf{v}, \nabla \cdot \mathbf{s})+(\mathbf{v}, \nabla p)+(\mathbf{v}, \mathbf{f}) & =0 & & \forall \mathbf{v} \\
(q, \nabla \cdot \mathbf{u})-\left(q, \frac{1}{K} p\right) & =0 & & \forall q
\end{aligned}
$$

where $\mathbf{v} \in \mathcal{V}$ and $q \in \mathcal{Q}$ are the variations of the displacements and pressure fields, respectively, and $(\cdot, \cdot)$ denotes the inner product in $L^{2}(\Omega)$, the space of square integrable functions in $\Omega$. Integrating Eq. (13) by parts, the problem can be rewritten in the standard form as:

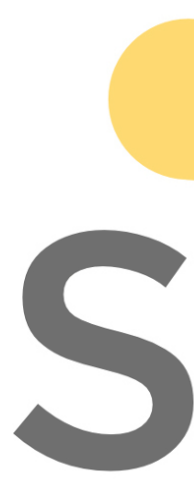

$$
\left(\nabla^{s} \mathbf{v}, \mathrm{s}\right)
$$

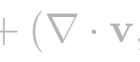

$$
\begin{aligned}
& , p)-(\mathbf{v}, \mathbf{f})-(\mathbf{v}, \overline{\mathrm{t}})_{\partial \Omega}=0 \quad \forall \mathbf{v} \\
& (q, \nabla \cdot \mathbf{u})-\left(q, \frac{1}{K} p\right)=0 \quad \forall q
\end{aligned}
$$
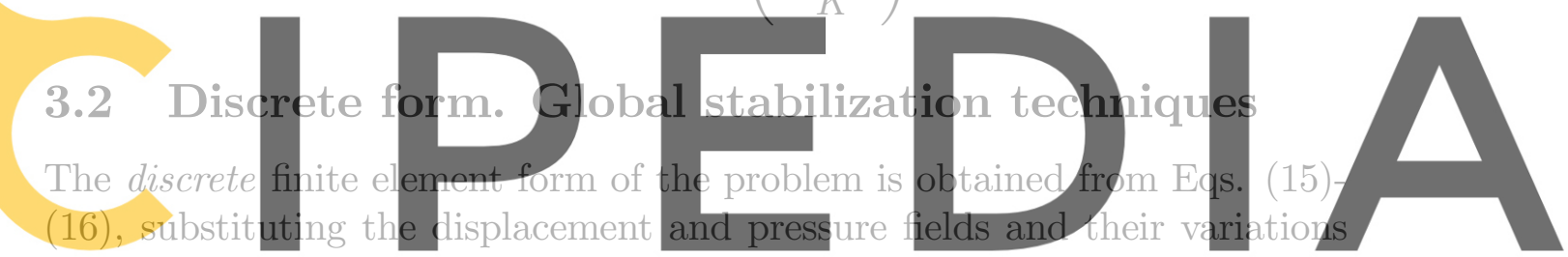

by their standard finite element interpolations:

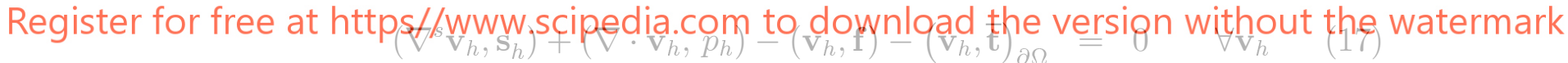

$$
\left(q_{h}, \nabla \cdot \mathbf{u}_{h}\right)-\left(q_{h}, \frac{1}{K} p_{h}\right)=0 \quad \forall q_{h}
$$

where $\mathbf{u}_{h}, \mathbf{v}_{h} \in \mathcal{V}_{h}$ and $p_{h}, q_{h} \in \mathcal{Q}_{h}$ are the discrete displacement and pressure fields and their variations, defined onto the finite element spaces $\mathcal{V}_{h}$ and $\mathcal{Q}_{h}$, respectively. As it is well known, the BB-condition [29], poses severe restrictions on the choice of the spaces $\mathcal{V}_{h}$ and $\mathcal{Q}_{h}$ when using the standard Galerkin discrete form (17)-(18). For instance, standard mixed elements with continuous equal order linear/linear interpolation for both fields are not stable, and the lack of stability shows as uncontrollable oscillations in the pressure field that usually, and very particularly in non linear problems, pollute the solution entirely. Fortunately, the strictness of the BB-condition can be circumvented by modifying the discrete variational form appropriately, 
in order to attain the necessary global stability with the desired choice of interpolation spaces.

One way of "expanding" the choice of interpolation spaces comes from the sub-grid scale approach, firstly proposed in [21]. The concept was further exploited in [24], where the concept of orthogonal sub-scales was introduced and, thereafter, successfully applied to several fluid dynamics problems. Application of the orthogonal sub-grid scale stabilization method (OSGS) to the problem of incompressible elasto-plasticity has been formulated by the authors in previous works and the interested reader is referred to them for a detailed explanation, see [25], [26] and [27].

The basic idea is to "enhance" the approximation of the discrete displacements provided from the "coarse" finite element grid with the stabitization effect of a finer "sub-grid" scale. The displacements of the sub-scale are thought of a perturbation of the ones resolved by the finite element scale. By definition, this sub-grid component cannot be resolved by the computational grid, but appropriate approximations can be introduced in order to account for their desired effects. With these approximations it is possible to
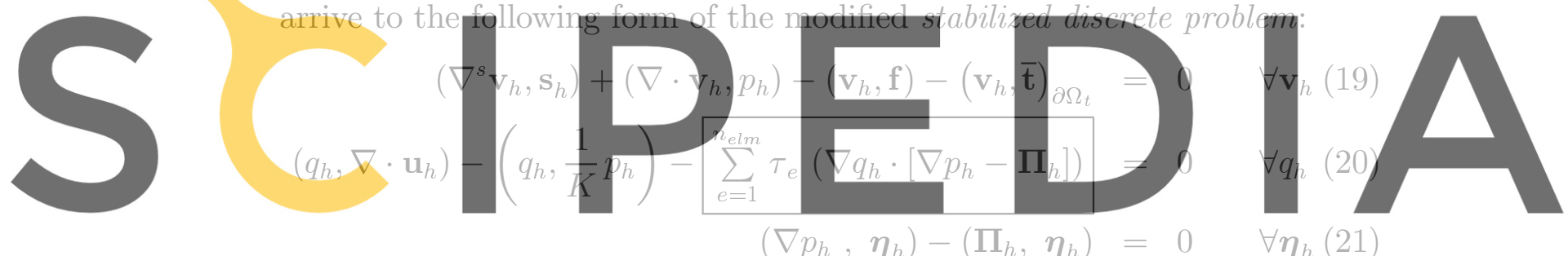

$$
\left(\nabla p_{h}, \eta_{h}\right)-\left(\Pi_{h}, \eta_{h}\right)=0
$$

$\forall \boldsymbol{\eta}_{h}(21)$

Register for free at https//Www scipedia.com to download the version without the watermark the characteristic length of the element $h_{e}$ and the shear modulus $G$. The constant $c=\mathcal{O}(1)$ has to be determined through numerical testing.

It is important to point out that, when using linear/linear displacement and pressure interpolations, with this approach no stabilization term appears in the momentum balance equation (19), which can be solved as in a standard mixed finite element formulation. The only stabilization term appears in the incompressibility equation (20). Observe that in it, a third nodal variable $\Pi_{h}$ is involved. As can be seen in Eq. (21), this is not other that the $L_{2-}$ projection (least square fitting) of the pressure gradient, $\mathbf{\Pi}_{h}=P_{h}\left(\nabla p_{h}\right)$. The next section shows that the drawback of accounting for an extra nodal variable can be easily overcome to achieve a robust and efficient procedure.

It is obvious in Eq. (20) that the effect of the stabilization technique is to add a term that relaxes the strict quasi-incompressibility constraint posed 
by this equation. Note that this relaxation is very small, as $\tau_{e}=\mathcal{O}\left(h^{2}\right)$ and it depends on the difference between the discontinuous pressure gradient and its continuous least square projection. This difference tends to vanish as the mesh is refined.

An alternative, somewhat simpler, stabilization method is the one known as Galerkin Least Square (GLS), originally proposed in [30] and [31], and used in solid mechanics in [13] to solve finite strain elasticity problems. The corresponding stabilized discrete problem reads:

$$
\begin{aligned}
\left(\nabla^{s} \mathbf{v}_{h}, \mathbf{s}_{h}\right)+\left(\nabla \cdot \mathbf{v}_{h}, p_{h}\right)-\left(\mathbf{v}_{h}, \mathbf{f}\right)-\left(\mathbf{v}_{h}, \overline{\mathbf{t}}\right)_{\partial \Omega_{t}} & =0 \\
\left(q_{h}, \nabla \cdot \mathbf{u}_{h}\right)-\left(q_{h}, \frac{1}{K} p_{h}\right)-\sum_{e=1}^{n_{\text {elm }}} \tau_{e}\left(\nabla q_{h} \cdot \nabla p_{h}\right) & =0 \quad \forall \mathbf{v}_{h}
\end{aligned}
$$

which has a format very similar to the OSGS method, but does not require the computation of any extra nodal variable. Experience shows that the GLS method is more "diffusive" that the OSGS stabilization. This means that GLS is somewhat more "robust" than OSGS, but sometimes less sharp localizations are obtained.

\subsection{Local stabilization}

The global stabilization techniques discussed in the previous section are designed to provide global stability to the incompressible elasto-plastic problem. In the cases of elastic incompressibility or smooth widespread, non-localizing, plastic responses, they are able to preclude the pressure oscillations that arise if the standard Galerkin method is used (see [25] and [26]). In reference [27] it was found that, with perfect plasticity, the stabilization parameter has to be modified to account for the development of the plastic regime, as deformation localizes into weak discontinuities; otherwise, pressure oscillations arise in the vicinity of the plastic areas that pollute the solution.

In [27], it was proposed to enhance the stabilization properties using a nonlinear stabilization parameter, $\tau_{e}=c h_{e}^{2} / 2 G^{*}$, computed as a function of the characteristic length of the element $h_{e}$ and the current secant shear modulus $G^{*}$, defined as (half) the ratio between the norms of the deviatoric stress and total strain tensors, $2 G^{*}=\left\|\mathbf{s}_{h}\right\| /\left\|\mathbf{e}_{h}\right\|$. For plasticity, this ratio is obviously non-constant and it varies along the deformation process. It is clear that, as plastic deformation evolves, the ratio $G^{*} / G$ decreases and, 
consequently, the value of $\tau_{e}$ increases, further relaxing the incompressibility constraint imposed by the isochoric nature of the plastic flow.

\subsection{Consistent residual viscosity}

For softening materials, strain localizes and the ratio $G^{*} / G$ decreases very fast with plastic deformation and, ultimately, vanishes, yielding very large values of the stabilization parameter. In many practical applications it is found that this almost unbounded increase of the stabilization term causes numerical difficulties that translate in slow or even lack of convergence of the solution of the nonlinear process, particularly in problems involving singular points, where the strains reach very high values in early stages of the analysis.

One obvious way to alleviate this difficulty is to define an ad hoc cut-off for the decrease of the values of the secant modulus $G^{*}$ or, alternatively, on the increase of the values of the stabilization parameter $\tau_{e}$. Unfortunately, experience shows that this leads to the impossibility of the total elimination of the local oscillations of the pressure. The greater the value of the cut-off, the better is the convergence, but greater are the remaining local pressure oscillations.

Therefore, a different approach is preferred to enhance the convergence of the nonlinear equilibrium iterations. This consists in introducing a viscous regularization of the plastic model, transforming it in (slightly) viscoplastic. The numerical benefits of this viscous regularization are well known and documented in the literature, but it is also true that the addition of "artificial" viscosity in an indiscriminate fashion rather changes the nature of the obtained solution. It is far more elegant to devise a high order scheme where the minimal amount of artificial viscosity is added only where it is needed and, also, in a consistent manner, that is, with smaller values for decreasing element sizes.

It is here proposed to regularize the elasto-plastic constitutive model with an artificial viscosity defined in terms of the the normal proyection of the residual of the momentum equation onto the finite element space (see [27]), in the form

$$
\eta=c^{\prime} h_{e} \Delta t\left\|\nabla p_{h}-\Pi_{h}\right\|
$$

where $c^{\prime}$ is a constant, $h_{e}$ is the characteristic length of the element and $\Delta t$ is the time step size. Note that this viscosity acts only in those elements where the momentum equation is not exactly satisfied and that, for linear 
simplex, it is $\eta=\mathcal{O}\left(h_{e}^{2} \Delta t\right)$. This means that it maintains the order of the finite element approximation, as it vanishes upon mesh (and time increment) refinement with the appropriate rate.

The structure of expression (24) suggests that it is also possible to define the viscosity purely in terms of the norm of the pressure gradient, in the form

$$
\eta=c^{\prime} h_{e} \Delta t\left\|\nabla p_{h}\right\|
$$

This second proposal is consistent with the definition of the stabilization term used in the GLS method.

\section{Implementation and computational aspects}

Due to the nonlinear dependence of the stresses on the displacements, the solution of the system of equations (19)-(21) requires the use of an appropriate incremental/iterative procedure such as the Newton-Raphson method. Within such a procedure, the system of linear equations to be solved for the $(i+1)$-th equilibrium iteration of the $(n+1)$-th time (or load) step is:

$$
\left[\begin{array}{ccc}
\mathbf{K}_{\mathrm{dev}} & \mathbf{G} & \mathbf{0} \\
\mathbf{G}^{T} & -\left(\frac{1}{K} \mathbf{M}+\mathbf{L}_{\boldsymbol{\tau}}\right) & \mathbf{G}_{\boldsymbol{\tau}}^{T} \\
\mathbf{0} & \mathbf{G}_{\boldsymbol{\tau}} & -\mathbf{M}_{\boldsymbol{\tau}}
\end{array}\right]^{(n+1, i)}\left[\begin{array}{l}
\delta \mathbf{U} \\
\delta \mathbf{P} \\
\delta \mathbf{\Pi}
\end{array}\right]^{(n+1, i+1)}=-\left[\begin{array}{l}
\mathbf{R}_{1} \\
\mathbf{R}_{2} \\
\mathbf{0}
\end{array}\right]^{(n+1, i)}
$$

where $\delta \mathbf{U}, \delta \mathbf{P}$ and $\delta \boldsymbol{\Pi}$ are the iterative corrections to the nodal values for the displacements, pressure and pressure gradient, respectively, $\mathbf{R}_{1}$ and $\mathbf{R}_{2}$ are the residual vectors associated to the satisfaction of the balance of momentum and incompressibility equations, respectively, and the global matrices $\mathbf{K}_{\mathrm{dev}}, \mathbf{G}, \mathbf{G}_{\tau}, \mathbf{M}, \mathbf{L}_{\tau}$ and $\mathbf{M}_{\tau}$ come from the standard assembly procedure of the elemental contributions, for the $i$-th iteration of the $n+1$ step. Note that this global matrix is symmetric, but it is not positive definite.

The monolithic solution of system (26) can be avoided by using a staggered procedure, in which the pressure projection $\Pi^{(n+1, i+1)}$ is solved independently and explicitly. To this end, from the third equation, it is possible to express $\boldsymbol{\Pi}^{(n+1, i+1)}$ in terms of $\mathbf{P}^{(n+1, i+1)}$ as:

$$
\boldsymbol{\Pi}^{(n+1, i+1)}=\left(\mathbf{M}_{\tau}^{-1}\right)^{(n+1, i)} \mathbf{G}_{\boldsymbol{\tau}}{ }^{(n+1, i)} \mathbf{P}^{(n+1, i+1)} \cong \overline{\mathbf{M}}^{-1} \mathbf{G} \mathbf{P}^{(n+1, i+1)}
$$

The computation of the projections $\Pi$ can be transformed in a straightforward operation by neglecting the difference in the $\tau_{e}$ coefficient in adjacent elements and considering an approximate lumped mass matrix $\overline{\mathbf{M}}$. 
One further approximation can be introduced to make the solution of the mixed system of equations more efficient from the computational point of view. This consists in keeping the projected pressure gradient constant during the equilibrium iterations within each time increment, taking it equal to the corresponding value at the end of the previous time step, that is $\boldsymbol{\Pi}^{(n+1, i+1)} \cong \boldsymbol{\Pi}^{(n)}$. This strategy has proved effective without loss of precision nor robustness.

From the above, the implementation of the GLS method is straightforward; it is enough to delete the third equation in (26), together with the corresponding third column of the global matrix, to yield:

$$
\left[\begin{array}{cc}
\mathbf{K}_{\mathrm{dev}} & \mathbf{G} \\
\mathbf{G}^{T} & -\left(\frac{1}{K} \mathbf{M}+\mathbf{L}_{\boldsymbol{\tau}}\right)
\end{array}\right]^{(n+1, i)}\left[\begin{array}{l}
\delta \mathbf{U} \\
\delta \mathbf{P}
\end{array}\right]^{(n+1, i+1)}=-\left[\begin{array}{l}
\mathbf{R}_{1} \\
\mathbf{R}_{2}
\end{array}\right]^{(n+1, i)}
$$

where the effect of the stabilization matrix $\mathbf{L}_{\tau}$ is evident. For the OSGS, the stabilization matrix can be formally expressed as $\widehat{\mathbf{L}}_{\tau}=\mathbf{L}_{\tau}-\mathbf{G}_{\tau}^{T} \mathbf{M}_{\tau}^{-1} \mathbf{G}_{\tau}$.

\section{Numerical results}

The formulation presented in the preceding sections is illustrated below in a number of benchmark problems. Performance of the global and local stabilization method is tested considering $2 \mathrm{D}$ plane-strain triangular meshes and 3D tetrahedral meshes. The examples involve both compressible and incompressible elasticity and J2-plasticity with exponential softening. The following material properties are assumed: Young's modulus $E=10 \mathrm{MPa}$, different Poisson's ratios (recall that $G=E / 2(1+\nu), K=E / 3(1-2 \nu)$ ), yield stress $\sigma_{o}=E / 1000=10 \mathrm{KPa}$ and different fracture energies. A value $c=1$ is taken for the evaluation of $\tau_{e}$. Values in the range $c^{\prime}=10 \div 100$ are used for the evaluation of the viscous regularization.

The Newton-Raphson method, combined with a line search procedure is used to solve the non-linear system of equations arising from the spatial and temporal discretization of the weak form of the stabilized problem. In all cases 200 equal time steps are performed to complete the analyses. Calculations are performed with an enhanced version of the finite element program COMET [32], developed by the authors at the International Center for $\mathrm{Nu}$ merical Methods in Engineering (CIMNE). Pre and post-processing is done with GiD, also developed at CIMNE [33]. 


\subsection{D Perforated strip}

The first example is a plane-strain perforated strip loaded through a rigid platen which sustains an axial central point load. Because of the double symmetry, only one quarter of the domain (the top right quarter) needs to be discretized. Figure 2a depicts the geometry of the problem; dimensions are related to length $r=1 \mathrm{~m}$. Figure 2 also shows two of the three unstructured meshes used in the analyses: (b) a coarse mesh of 438 linear triangles (251 nodes), (c) a medium mesh of 1,903 linear triangles (1,020 nodes), and (not shown) a fine mesh of 7,489 linear triangles (3,880 nodes). Notice that in all the unstructured meshes the pre-processor used tends to introduce patches or equilateral triangles with predominant directions at $-30^{\circ},+30^{\circ}$ and $+90^{\circ}$.

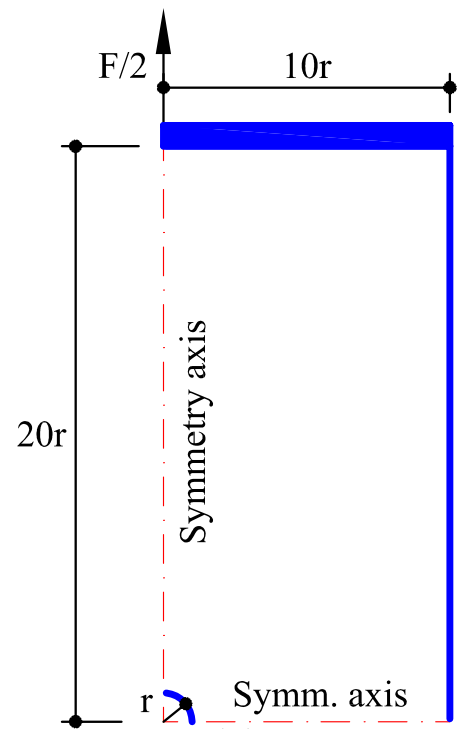

(a)

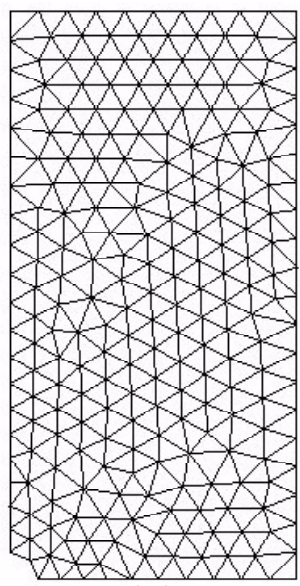

(b)

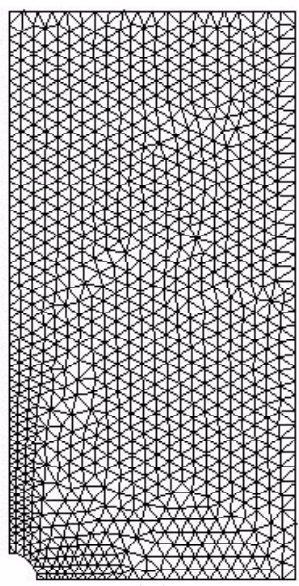

(c)

Figure 2: Geometry and meshes for the 2D perforated strip: (a) geometry, (b) coarse mesh, (c) medium mesh

For this example, a Poisson's ratio $\nu=0.3$ is used and, therefore, compressible elasticity is assumed; fracture energy is $G_{f}=200 \mathrm{~J} / \mathrm{m}^{2}$ (which corresponds to a softening parameter $\bar{H}_{S}=0.25 \mathrm{MPa} / \mathrm{m}$ ). The value $c^{\prime}=10$ is used for the evaluation of the viscous regularization.

Figure 3 shows (half)-load vs ((half)-imposed point of application)-displacement curves ( $1 \mathrm{~m}$ thickness is assumed) obtained with different formulations: (a) standard irreductible, (b) standard mixed, (c) mixed with global OSGS sta- 


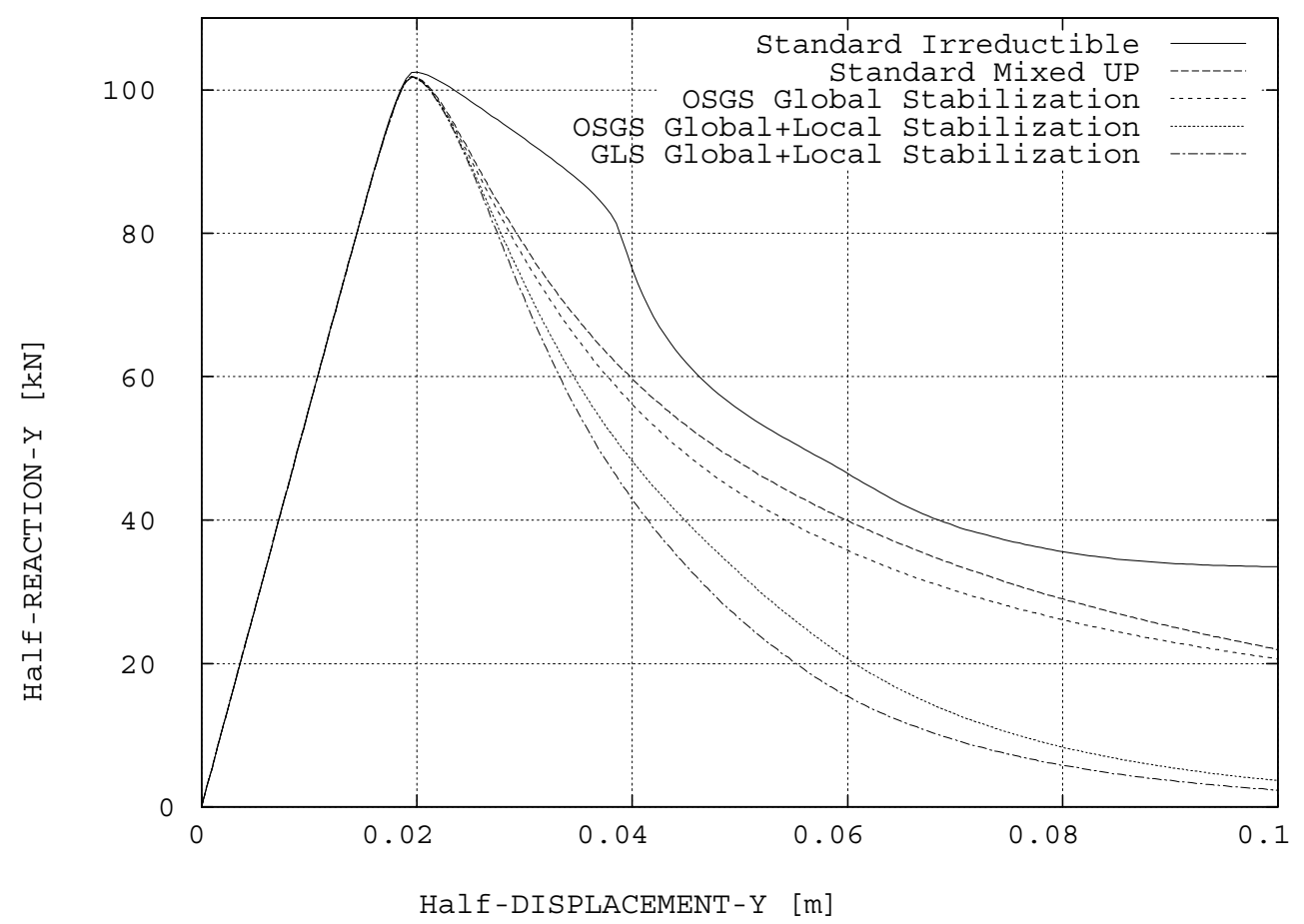

Figure 3: (Half)-Load vs (half)-imposed displacement curve for 2D perforated strip using different formulations

bilization, (d) mixed with global and local OSGS stabilization, and (e) mixed with global and local GLS stabilization. The corresponding analyses are run using the medium mesh depicted in Figure 2c. Several remarks are in order. First, all the mixed formulations capture reasonably well both the limit load and the general softening trend of the curve, while the irreductible formulation fails to do so almost completely. Second, the effect of the stabilization techniques adopted is obvious in the global softening response, with clear advantage for the global and local method over the purely global one. Third, the difference between the OSGS and the GLS methods is small. As it will be seen below, the differences in the responses are due to the different degrees of success of the formulations in removing the volumetric locking induced by the development of the plastic behaviour.

Figures 4 and 5 depict the deformed shape and contours of the effective plastic strain once the plastic flow is fully developed and the collapse 


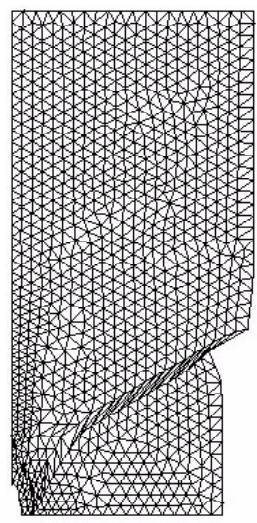

(a)

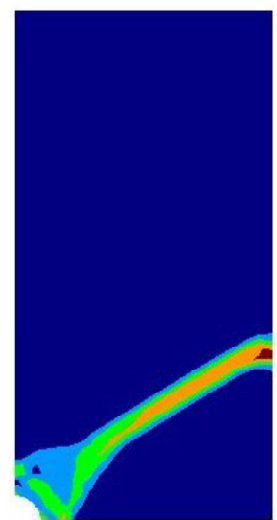

(b)

Figure 4: Deformed shape and equivalent plastic strain contour fills for the 2D perforated strip with irreductible formulation

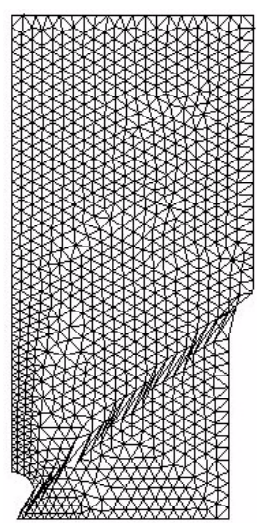

(a)

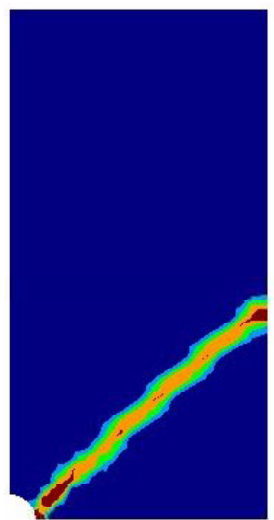

(b)

Figure 5: Deformed shape and equivalent plastic strain contour fills for the 2D perforated strip with stabilized mixed formulation

mechanism can be appreciated (vertical displacement of the load $\delta=0.1$ $\mathrm{m}$ ), both for the irreductible and the stabilized mixed formulations. On one hand, Figure 4 shows results for the irreductible formulation. Note how the localization band forms an angle of $+30^{\circ}$ with the horizontal axis and it is completely influenced by the directional bias of the mesh.

Correspondingly, Figure 5 shows results for the stabilized mixed formulation. Here, the localization band forms a correct angle of approximately $+45^{\circ}$ with the horizontal axis and it is virtually free on any directional bias from 


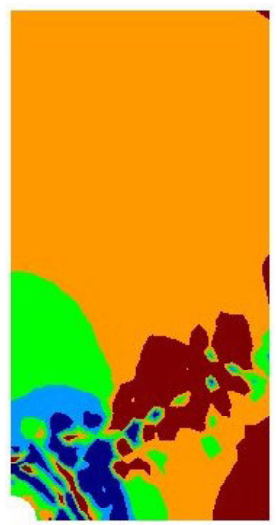

(a)

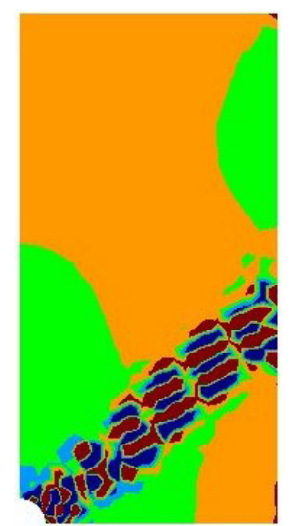

(b)

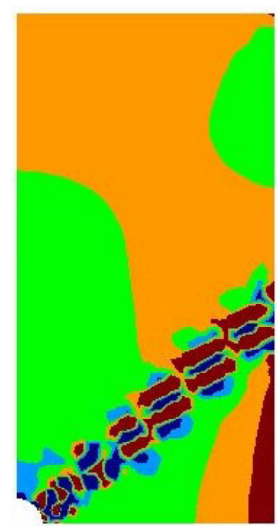

(c)

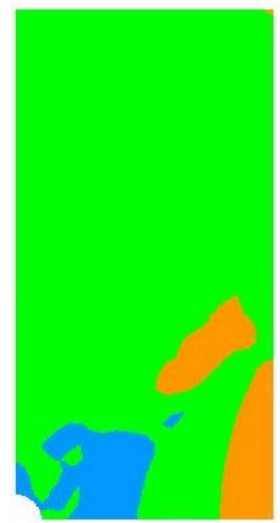

(d)

Figure 6: Pressure contour fills for the 2D perforated strip with different formulations: (a) irreductible, (b) standard mixed, (c) OSGS with global stabilization, (d) OSGS with global and local stabilization

the mesh. It has to be remarked that the deformation pattern and collapse mechanism predicted by all the mixed formulations are practically identical, independently of the stabilization method used, or even if no stabilization is introduced. Therefore, the ability of the mixed formulation to capture the correct plastic localization pattern is demonstrated.

Figure 6 presents pressure contours at the same time (final) of the deformation process. Lack of stability and severe oscillations of the pressure field can be identified in both the irreductible (a) and mixed (b) standard formulations. This is enough to completely destabilize the irreductible formulation, although for this example it seems to have little influence in the deformation pattern of the mixed formulation, as the plastic deformation does not depend on the actual values of the pressure. Obviously, this is not always necessarily the case, and especially in nonlinear analyses. Also, the improved performance of the stabilized formulations is easily perceptible. Note that if a linear stabilization term is used, (c), only global stability is achieved and pressure oscillations are still visible in the vicinity of the localization band, both along and across the direction of the slip. This oscillations are completely removed from figure (d), where the nonlinear stabilization parameter is used, achieving both global and local stabilization of the pressure field. 


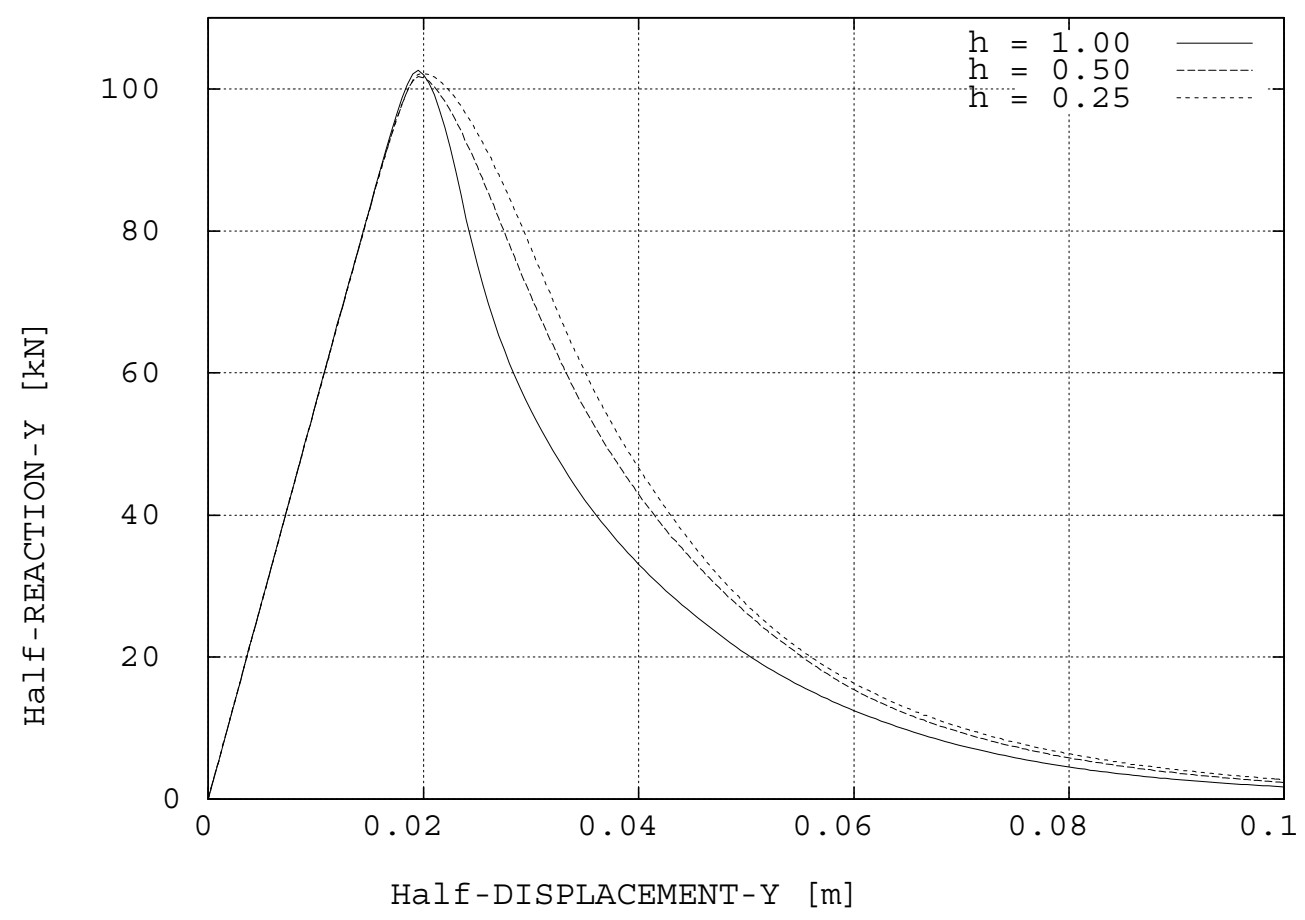

Figure 7: (Half)-Load vs (half)-imposed displacement curve for 2D perforated strip using different meshes

Finally, Figure 7 shows (half)-load vs ((half)-imposed point of application)displacement curves (1m thickness is assumed) obtained with different unstructured meshes: (a) coarse, (b) medium, and (c) fine. Note that the overall global response is satisfactorily convergent upon mesh refinement, with the total area under the load-displacement curve converging to the correct amount of plastic work dissipated to create the localization band. No spurious brittleness is observed when the size of the elements is reduced. This is achieved by the regularization procedure explained in Section 2.2.

\subsection{D Prandtl's punch test}

The second example is the Prandtl's punch test, a well-known plane-strain 2D problem often used in the literature to test the ability of J2-plastic models to capture collapse loads and mechanisms. Figure 8a depicts the geometry of 


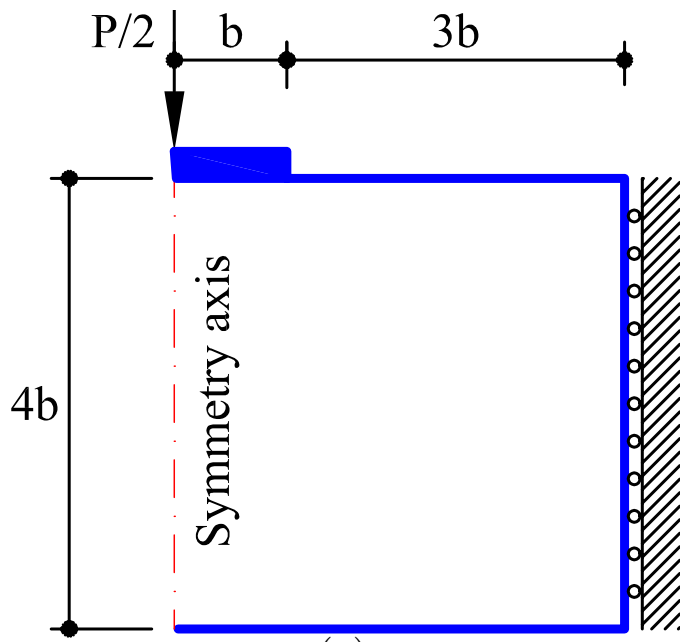

(a)

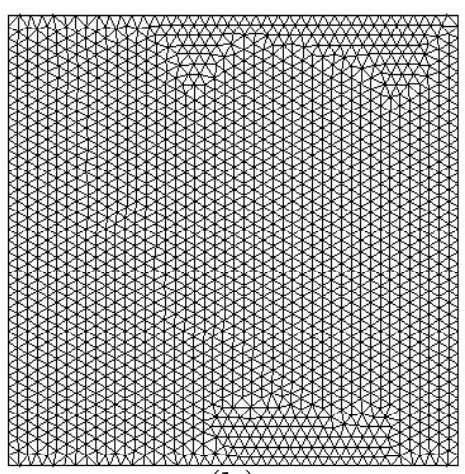

(b)

Figure 8: Geometry (a) and mesh (b) used for the 2D Prandtl's punch test

the problem, a rigid footing with a central point load; dimensions are related to length $b=1 \mathrm{~m}$.

The elastic behaviour is assumed to be incompressible and, therefore, a Poisson's ratio $\nu=0.5$ is used in the analyses. Nevertheless, when using the irreductible formulation a reduced value $\nu=0.45$ had to be used to obtain results, even if they were very inaccurate. Fracture energy is $G_{f}=50 \mathrm{~J} / \mathrm{m}^{2}$ (which corresponds to a softening parameter $\bar{H}_{S}=1 \mathrm{MPa} / \mathrm{m}$ ). The value $c^{\prime}=100$ is used for the evaluation of the viscous regularization.

Because of the symmetry, only half of the domain (the right half) needs to be discretized. The mesh used in the computations is shown in Figure 8b, unstructured with 3,624 linear triangles and 1,893 nodes. It has to be remarked that experience, and the previous example, show that localization problems require the use of much finer meshes than those necessary for problems were displacements and strains do not localize.

Figure 9 shows (half)-load vs (point of application)-displacement curves (1 $\mathrm{m}$ thickness is assumed) obtained with different formulations: (a) standard irreductible, (b) standard mixed, (c) mixed with global OSGS stabilization, (d) mixed with global and local OSGS stabilization, and (e) mixed with global and local GLS stabilization. The results confirm the observations of the previous example: the irreductible formulation locks almost completely, while the other three formulations yield meaningful softening curves. Again, 


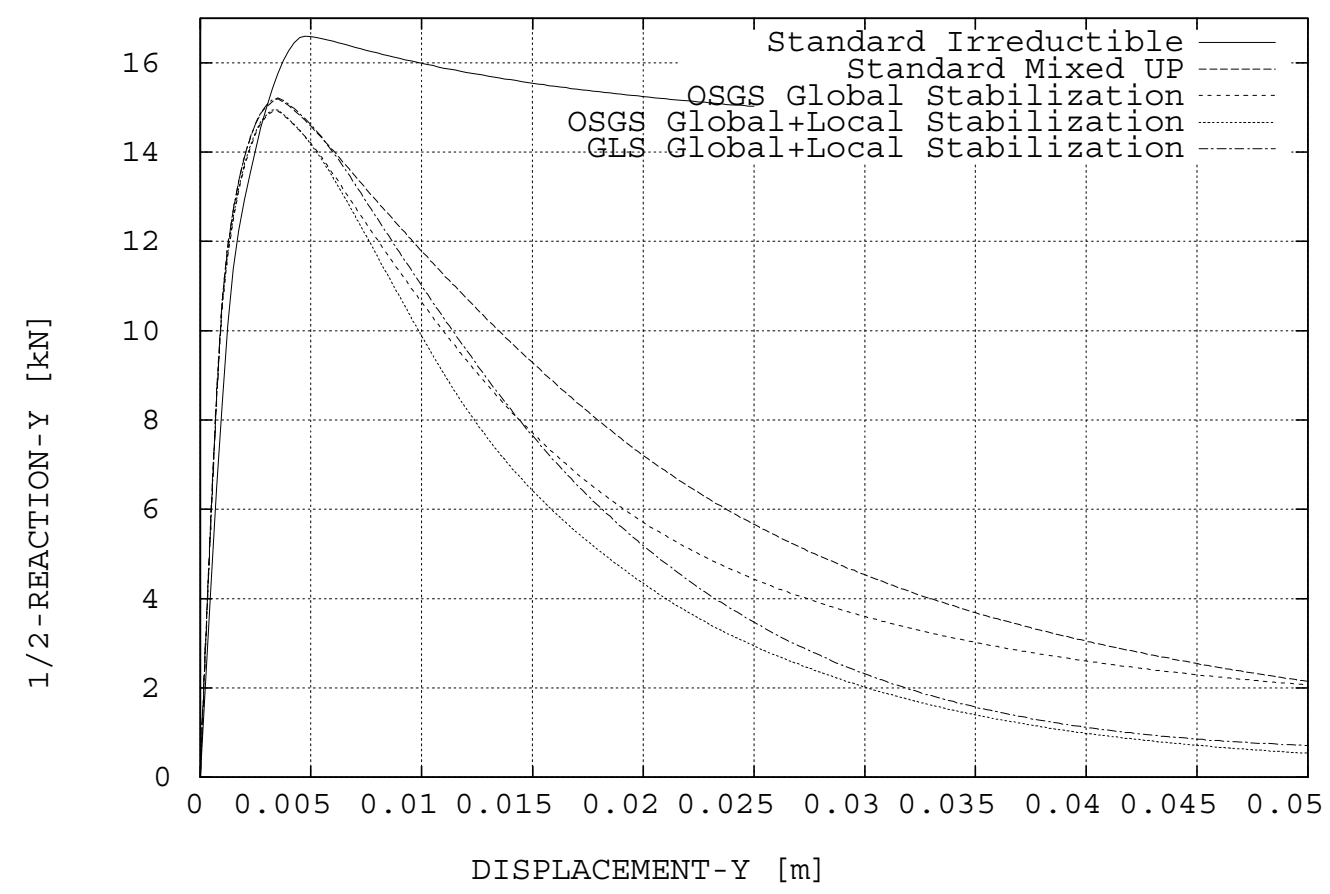

Figure 9: (Half)-load-displacement curve for 2D Prandtl's punch test

as it will be seen below, the differences in the mixed responses are due to the variable degrees of success of the various formulations in removing the volumetric locking induced by the plastic behaviour.

Figures 10 and 11 depict the deformed shape and contours of the effective plastic strain once the plastic flow is fully developed and the collapse mechanism can be appreciated (vertical displacement of the load $\delta=0.05$ $\mathrm{m})$, both for the irreductible and the stabilized mixed formulations. On one hand, Figure 10 shows results for the irreductible formulation. Note how the collapse mechanism is completely influenced by the directional bias of the mesh, with slip bands that follow exactly the predominant mesh orientations at $-30^{\circ},+30^{\circ}$ and $+90^{\circ}$. Also spurious localization branches appear.

Correspondingly, Figure 11 shows results for the stabilized mixed formulation. Note how here, the localization bands form according to the well-known classical solution (for rigid-perfectly plastic behaviour) and it is virtually free on any directional bias from the mesh. It is particularly remarkable how the expected logarithmic spiral branch, with no spurious branching, is ob- 


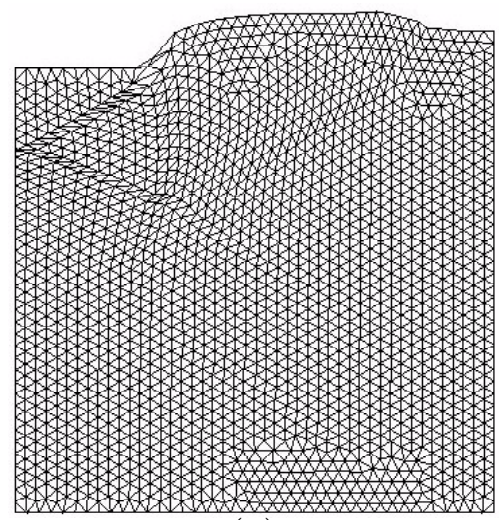

(a)

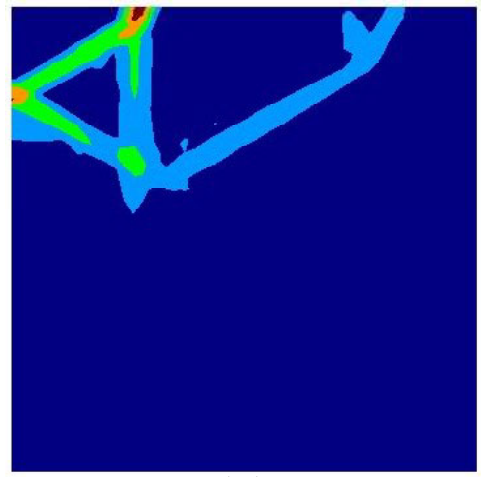

(b)

Figure 10: Deformed shape and equivalent plastic strain contour fills for the 2D perforated strip with irreductible formulation

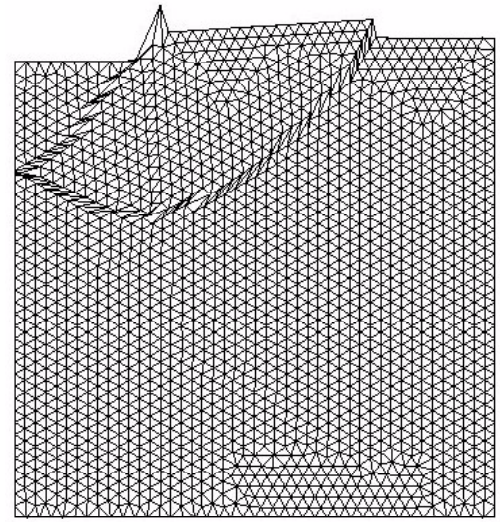

(a)

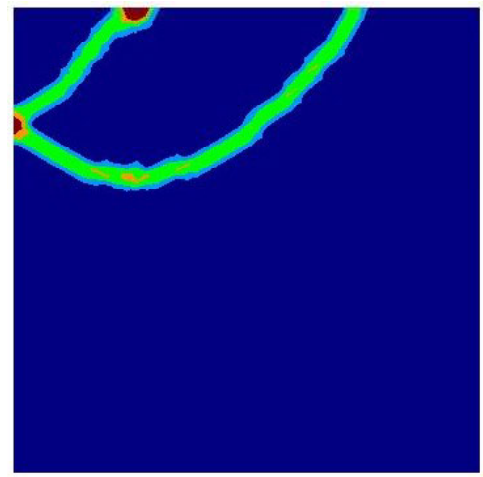

(b)

Figure 11: Deformed shape and equivalent plastic strain contour fills for the 2D perforated strip with stabilized mixed formulation

tained. Again, the deformation pattern and collapse mechanism predicted by all the mixed formulations are practically identical, independently of the stabilization method used, which demonstrates the superiority of the mixed formulation for capturing the correct plastic localization pattern.

Finally, Figure 12 presents pressure contours at the same time (final) of the deformation process, where the serious deficiencies of the standard formulations and the huge improvement achieved with a proper stabilization are evident. Note again how the proposed stabilization technique in able to 


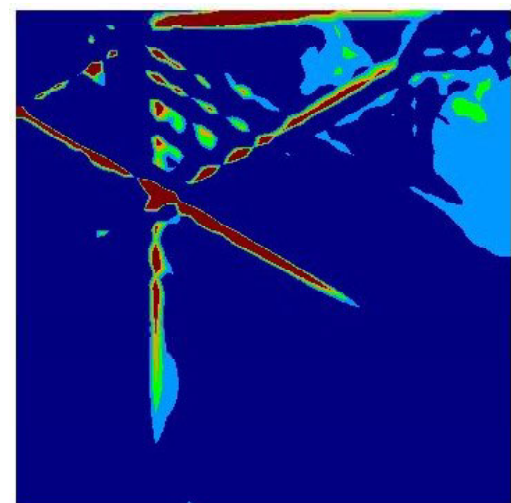

(a)

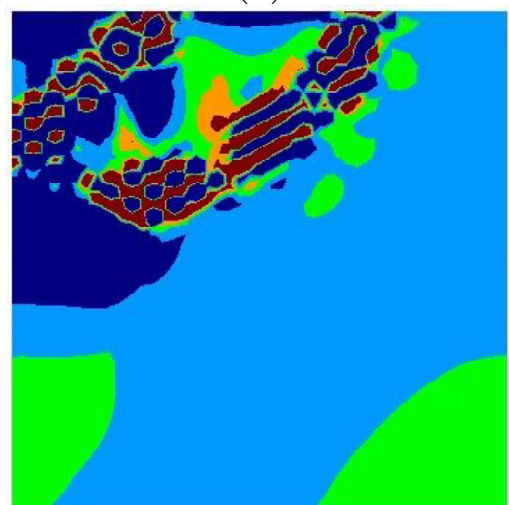

(c)

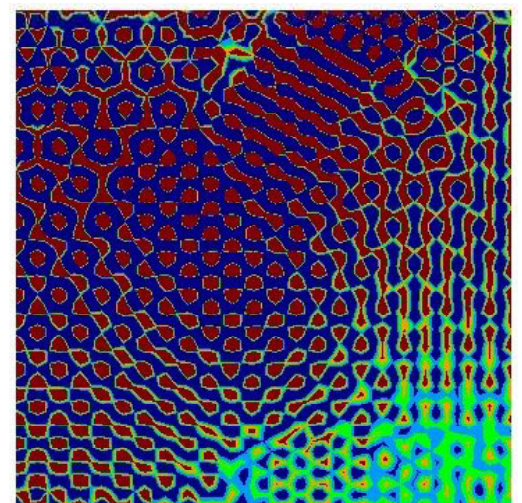

(b)

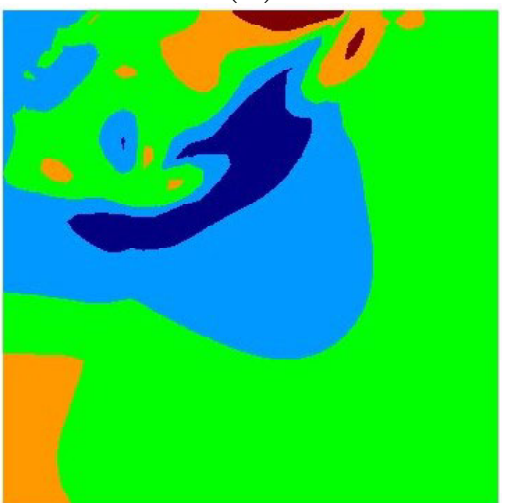

(d)

Figure 12: Pressure contour fills for the 2D perforated strip with different formulations: (a) irreductible, (b) standard mixed, (c) OSGS with global stabilization, (d) OSGS with global and local stabilization

eliminate completely the pressure oscillations, including those in the vicinity of the slip bands. 


\subsection{D Prandtl's punch test}

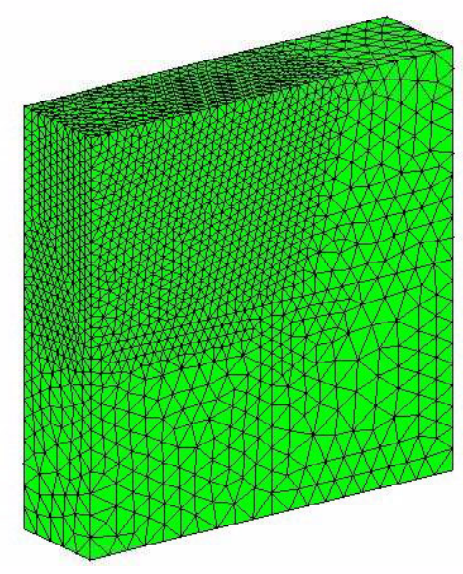

Figure 13: Unstructured mesh used for 3D Prandtl's punch test

A 3D version of the previous problem is presented next; Figure 13 shows the unstructured mesh of linear tetrahedra used in the computations $(56,514$ elements, 10,969 nodes). The thickness discretized is $b=0,5 \mathrm{~m}$. Note that the element sizes in the mesh are non-uniform, but the size of the element used in the part where the localization bands are expected to form is approximately the same used in the $2 \mathrm{D}$ mesh in the previous section. Roller supports are prescribed in the nodes on the frontal and rear faces to simulate plane strain conditions.

Figure 14 shows the (half)-load vs (point of application)-displacement curves obtained for the 2D and 3D cases, using the GLS method with global and local stabilization. It is remarkable that both cases yield such comparable results. The difference is attributed to the unstructured nature of the mesh in the transverse direction; this produces slip surfaces which are "rough" (and not perfectly cylindrical) in the transverse direction and, therefore, the plastic work developed is greater than in the $2 \mathrm{D}$ case.

Figure 15a displays contours of the effective plastic strain once the plastic flow is fully developed (vertical displacement of the load $\delta=0.05 \mathrm{~m}$ ). This figure matches closely the results obtained in the 2D case, depicted in Figure 11. It is therefore demonstrated that the proposed formulation can capture correctly the displacement and strain localization leading to the collapse 


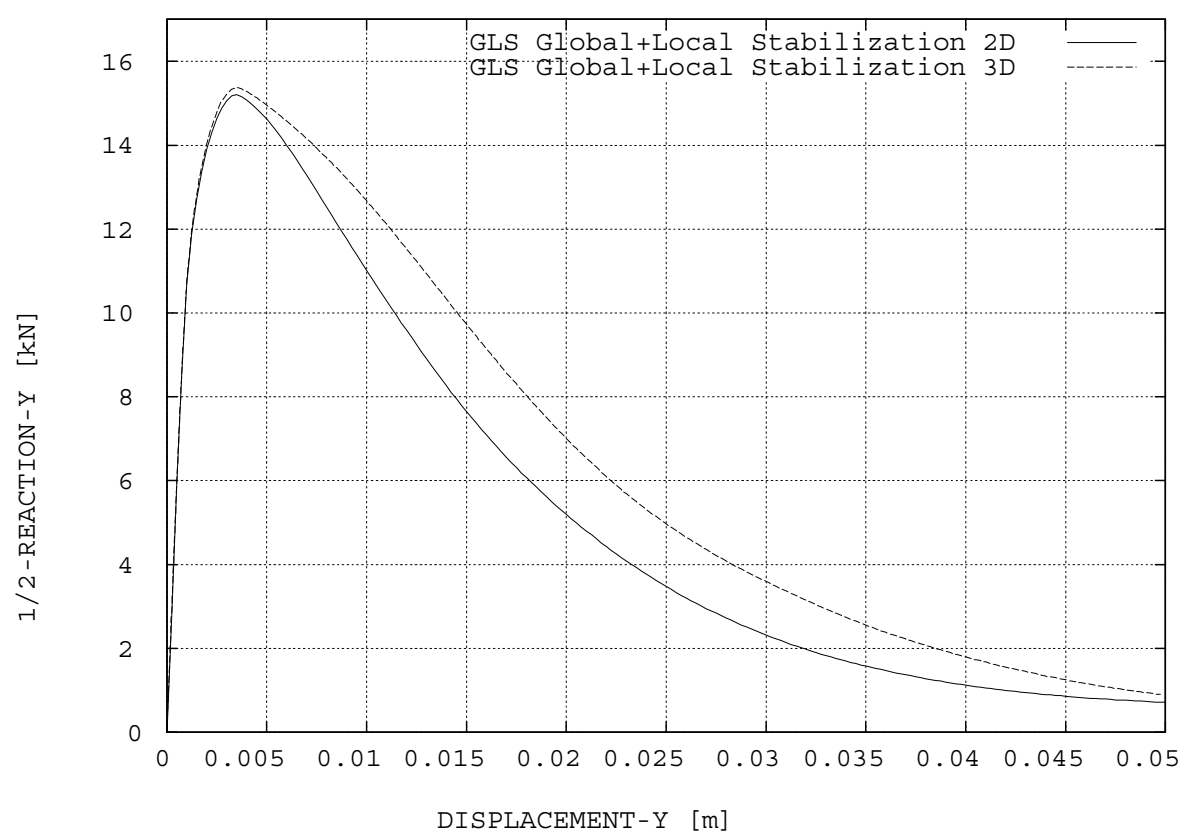

Figure 14: (Half)-Load-displacement curve for 2D and 3D Prandtl's punch test.

mechanism even in a $3 \mathrm{D}$ setting.

Finally, Figure 15b shows the pressure contours at the same time (final) of the deformation process. Again, it is remarkable that completely satisfactory results are obtained with the proposed formulation in an unstructured mesh of tetrahedra, very similar to those obtained in the $2 \mathrm{D}$ simulation.

\section{Conclusions}

This paper presents the application of stabilized mixed linear simplicial elements (triangles and tetrahedra) to the solution of problems involving the capture of discontinuous solutions in J2 plasticity. The proposed stabilization is based on the orthogonal sub-grid scales approach and it is designed to allow equal order interpolation of the displacement and pressure fields. A consistent viscous regularization is also proposed and successfully applied, which allows to overcome the convergence difficulties often encountered in 

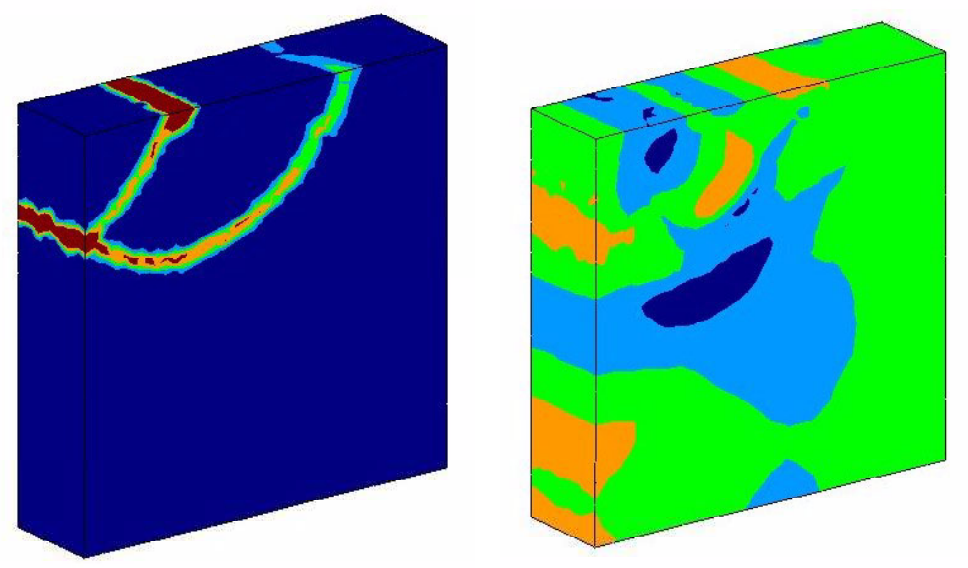

Figure 15: Equivalent plastic strain and pressure contour fills for 3D Prandtl's punch test with OSGS mixed formulations

applications involving softening behaviour and strain localization. The derived method yields a robust scheme, suitable for engineering applications in $2 \mathrm{D}$ and $3 \mathrm{D}$. The proposed stabilization scheme is shown to be enough to attain control on the pressure field, completely removing global and local oscillations induced by the incompressibility constraints induced by J2 plastic flow. Numerical examples show, on one hand, the tremendous advantage of the mixed formulation over the irreductible one to predict correct failure mechanisms with localized patterns of plastic deformation, which are practically free from any dependence of the mesh directional bias; on the other, stabilization techniques are shown to alleviate the volumetric locking exhibited by non-stable formulations, yielding an improved global response in the softening regime.

\section{Acknowledgments}

The authors gratefully acknowledge the inestimable help of Prof. R. Codina, expressed in the form of unfailing suggestions and discussions.

\section{References}

[1] de Borst, R., 2001. Some recent issues in computational failure mechan- 
ics. Int. J. Num. Meths. in Eng., 52, 63-95.

[2] de Borst, R., 2002. Fracture in quasi-brittle materials: a review of continuum damage-based approaches. Engineering Fracture Mechanics, 69, 95-112.

[3] Oliver, J. A consistent characteristic length for smeared cracking models. Int. J. Num. Meth. Engng., 28, 461-474.

[4] Oliver, J., Cervera, M. and Manzoli, O., 1999. Strong Discontinuities and Continuum Plasticity Models: The Strong Discontinuity Approach. Int. J. of Plasticity, 15, 1-34.

[5] Wells, G.N., Sluys, L.J. and de Borst, R. 2002. A p-adaptive scheme for overcoming volumetric locking during plastic flow. Comp. Meth. in Appl. Mech. and Eng., 191, 3153-3164.

[6] Zienkiewicz, O.C. and Taylor, R.L., 2000. The Finite Element Method, Butterworth-Heinemann, Oxford.

[7] Zienkiewicz, O.C., Pastor, M. and Huang, M., 1995. Softening, localization and adaptive remeshing: capture of discontinuous solutions. Comp. Mech., 17, 98-106.

[8] Zienkiewicz O.C., Huang, M. and Pastor, M., 1995. Localization problems in plasticity using finite elements with adaptive remeshing. Int. J. Num. Methods in Geomechanics, 19, 127-148.

[9] Pastor, M., Li, T., Liu, X. and Zienkiewcz, O.C., 1998. Stabilized linear triangles for failure problems in undrained soils. Proceedings of IV World Congress on Computational Mechanics: "Computational Mechanics: New Trends and Applications", S. Idelshon, E. Oñate and E. Dvorkin (eds.), CIMNE, Barcelona, Spain.

[10] Pastor, M., Li, T., Liu, X., Zienkiewcz, O.C. and Quecedo, M., 2000. A fractional step algorithm allowing equal order interpolation for coupled analysis of saturated soil problems. Mechanics of Cohesive-Frictional Materials, 5, 511-534.

[11] Bonet, J. and Burton, A.J., 1998. A simple average nodal pressure tetrahedral element for incompressible and nearly incompressible dynamic explicit applications. Comm. Num. Meths. in Eng., 14, 437-449. 
[12] Zienkiewicz, O.C., Rojek, J., Taylor, R.L. and Pastor, M., 1998. Triangles and tetrahedra in explicit dynamic codes for solids, Int. J. for Num. Meths. in Eng., 43, 565-583.

[13] Klaas, O., Maniatty, A. and Shephard, M.S., 1999. A stabilized mixed finite element method for finite elasticity. Formulation for linear displacement and pressure interpolation, Comp. Meth. in Appl. Mech. and Eng., 180, 65-79.

[14] Taylor, R.L. A mixed formulation for triangular and tetrahedral elements, In Abascal, R., Domínguez, J. and Bugeda, G., editors, Conference Proceedings on Métodos Numéricos en Ingeniería, SEMNI, Barcelona, Spain, 1999.

[15] Dohrmann, C.R., Heinstein, M.W., Jung, J., Key, S.W. and Witkowsky, W.R., 2000. Node-based uniform strain elements for three-node triangular and four-node tetrahedral meshes. Int. Jour. for Num. Meths. in Eng. 47, 1549-1568.

[16] Taylor, R.L., 2000. A mixed-enhanced formulation for tetrahedral elements. Int. Jour. for Num. Meths. in Eng. 47, 205-227.

[17] Bonet, J., Marriot, H. and Hassan, O., 2001. An averaged nodal deformation gradient linear tetrahedral element for large strain explicit dynamic applications. Comm. Num. Meths. in Eng., 17, 551-561.

[18] Bonet, J., Marriot, H. and Hassan, O., 2001. Stability and comparison of different linear tetrahedral formulations for nearly incompressible explicit dynamic applications. Int. Jour. for Num. Meths. in Eng., 50, 119-133.

[19] Oñate, E., Rojek, J., Taylor, R.L. and Zienkiewicz, O.C. Linear triangles and tetrahedra for incompressible problem usung a finite calculus formulation, Proceedings of European Conference on Computational Mechanics, ECCM, 2001.

[20] de Souza Neto, E.A., Pires, F.M.A. and Owen D.R.J. A new F-barmethod for linear triangles and tetrahedra in the finite strain analysis of nearly incompressible solids. Proceedings of VII International Conference on Computational Plasticity, COMPLAS, 2003. 
[21] Hughes, T.J.R., 1995. Multiscale phenomena: Green's function, Dirichlet-to Neumann formulation, subgrid scale models, bubbles and the origins of stabilized formulations, Comp. Meth. in Appl. Mech. and Eng., 127, 387-401.

[22] Codina, R. and Blasco, J., 1997. A finite element method for the Stokes problem allowing equal velocity-pressure interpolations, Comp. Meth. in Appl. Mech. and Eng., 143, 373-391.

[23] Hughes, T.J.R., Feijoó, G.R., Mazzei. L., Quincy, J.B., 1998. The variational multiscale method-a paradigm for computational mechanics, Comp. Meth. in Appl. Mech. and Eng., 166, 3-28.

[24] Codina, R., 2000. Stabilization of incompressibility and convection through orthogonal sub-scales in finite element methods, Comp. Meth. in Appl. Mech. and Eng., 190, 1579-1599.

[25] Chiumenti, M., Valverde, Q., Agelet de Saracibar, C. and Cervera, M., 2002. A stabilized formulation for incompressible elasticity using linear displacement and pressure interpolations, Comp. Meth. in Appl. Mech. and Eng., 191, 5253-5264.

[26] Chiumenti, M., Valverde, Q., Agelet de Saracibar, C. and Cervera, M., 2002. A stabilized formulation for incompressible plasticity using linear triangles and tetrahedra, submitted to Int. J. of Plasticity.

[27] Cervera, M., Chiumenti, M., Valverde, Q. and Agelet de Saracibar, C. 2003. Mixed Linear/linear Simplicial Elements for Incompressible Elasticity and Plasticity. Submitted to Comp. Meth. in Appl. Mech. and Eng.

[28] Simo, J.C. and Hughes, T.J.R. 1998. Computacional Inelasticity. Interdisciplinary Applied Mathematics. Vol. 7. Springer.

[29] Brezzi, F. and Fortin, M., 1991. Mixed and Hybrid Finite Element Methods, Spinger, New York.

[30] Hughes, T.J.R., Franca, L.P. and Balestra, M., 1986. A new finite element formulation for computational fluid dynamics: V. Circumventing the Babuska-Brezzi condition: A stable Petrov-Galerkin formulation 
of the Stokes problem accomodating equal-order interpolations. Comp. Meth. in Appl. Mech. and Eng., 59, 85-99.

[31] Hughes, T.J.R., Franca, L.P. and Hulbert, G.M., 1989. A new finite element formulation for computational fluid dynamics: VIII. The Galerkin/least-square method for advective-diffusive equations. Comp. Meth. in Appl. Mech. and Eng., 73, 173-189.

[32] Cervera, M., Agelet de Saracibar, C. and Chiumenti, M., 2002. COMET: COupled MEchanical and Thermal analysis. Data Input Manual, Version 5.0, Technical report IT-308, www.cimne.upc.es.

[33] GiD: the personal pre and post processor, 2002. www.gid.cimne.upc.es. 